\title{
Utilization of Colistin Versus $\beta$-Lactam and $\beta$-Lactamase Inhibitor Agents in Relation to Acute Kidney Injury in Patients with Severe Gram-Negative Infections
}

Casey Doremus $\cdot$ Stephen W. Marcella $\cdot$ Bin Cai $\cdot$ Roger M. Echols

Received: September 14, 2021 / Accepted: October 20, 2021 / Published online: November 3, 2021

(C) The Author(s) 2021

\section{ABSTRACT}

Introduction: Colistin is used to treat severe antibiotic-resistant Gram-negative infections (GNIs). With the rise of antibiotic resistance, colistin has been used increasingly as a 'lastline' therapy for multidrug-resistant GNIs. We evaluated the incidence of acute kidney injury (AKI) and mortality among patients receiving colistin or one of the new $\beta$-lactam $/ \beta$-lactamase inhibitors $(\beta \mathrm{L}+\beta \mathrm{LI})$ (ceftazidime/avibactam, ceftolozane/tazobactam, or meropenem/ vaborbactam).

Methods: This retrospective cohort study used data from the Premier Healthcare Database. The cohort included propensity score-matched adults with an inpatient stay between January 2016 and December 2018. Patients given both colistin and BL + BLI as treatment for $\geq 72 \mathrm{~h}$ were excluded. AKI was defined as acute renal failure or dialysis during hospitalization with antibiotic administration. Propensity score

Supplementary Information The online version contains supplementary material available at https:// doi.org/10.1007/s40121-021-00556-x.

C. Doremus · S. W. Marcella $(\varangle) \cdot$ B. Cai

Shionogi Inc, 300 Campus Drive, Florham Park, NJ 07932, USA

e-mail: stephen.marcella@shionogi.com

R. M. Echols

Infectious Disease Drug Development Consulting,

LLC, Easton, CT 06612, USA matching was used to control for selection bias and confounding. Logistic regression evaluated associations between treatment, AKI, and inhospital mortality.

Results: The total number of patients in the matched cohorts were 256 in each. Overall, $23.8 \%$ and $13.3 \%$ of patients receiving colistin or new $\beta \mathrm{L}+\beta \mathrm{LI}$ agents, respectively, experienced AKI during hospitalization $(p=0.002)$; odds of AKI for colistin were 3.0 (95\% CI 1.71, 5.21). Following propensity score-matching, patients without baseline renal disease experienced AKI during hospitalization to a higher degree in the colistin group compared to the $\beta \mathrm{L}+\beta \mathrm{LI}$ group $(17.1 \%$ vs. $6.8 \%)$; colistin use was associated with 3.7 times higher odds (95\% CI $1.84,7.42$ ) of AKI compared to $\beta \mathrm{L}+\beta \mathrm{LI}$ agents. The odds of mortality in patients on colistin developing AKI were more than three times that of patients receiving a BL + BLI agent who developed AKI. Among patients receiving colistin, incident AKI was associated with 6.1 times higher odds $(95 \%$ CI 2.53, 14.71) of mortality.

Conclusions: Patients receiving colistin for GNIs had significantly higher odds of AKI and mortality than those receiving $\beta \mathrm{L}+\beta \mathrm{LI}$.

Keywords: Colistin; $\beta$-lactam and $\beta$-lactamase inhibitor; Acute kidney injury; Mortality 


\section{Key Summary Points}

\section{Why carry out this study?}

Historically, colistin has been infrequently prescribed due to associated nephrotoxicity, but has recently been used more frequently for resistant pathogens, as it is the only generic antibiotic demonstrating reliable in vitro activity against some pathogens, such as A. baumannii.

Limited clinical experience of newer antibiotics to treat carbapenem-resistant Gram-negative infection (GNI) suggests a lower incidence of acute kidney injury (AKI).

The objectives of this study were to assess acute kidney injury and in-hospital mortality among patients receiving colistin or a new $\beta$-lactam/ $\beta$-lactamase inhibitor to treat a severe Gram-negative infection in a large population-based dataset.

\section{What was learned from the study?}

A large retrospective cohort study of the Premier Healthcare Database revealed that patients receiving colistin to treat Gramnegative infections had higher odds of mortality and acute kidney injury than those receiving a $\beta$-lactam/ $\beta$-lactamase inhibitor.

Utilizing newer, less toxic antibiotics to treat severe antibiotic-resistant Gramnegative infections could have significant implications for healthcare costs, morbidity, and mortality.

\section{INTRODUCTION}

Colistin is a polymyxin class antibiotic originally introduced into clinical practice in the 1950 s as one of the first antibiotics with significant efficacy for treating Gram-negative bacterial infections (GNIs) [1]. Due to reports of nephrotoxicity and neurotoxicity, historically, colistin was prescribed infrequently; thus, limited pharmacokinetic and pharmacodynamic data exist, and optimum dosing is ill-defined [2]. However, with the rise of antibiotic resistance, in particular carbapenem resistance (CR), colistin has been used increasingly as a 'lastline' therapy for multidrug-resistant (MDR) GNIs, including carbapenem-resistant Enterobacterales (CRE), Pseudomonas aeruginosa, and Acinetobacter baumannii (CRAB) [3]. MDR $P$. aeruginosa and CRAB, along with CRE, have, respectively, been identified by the $\mathrm{CDC}$ as serious and urgent threats [4].

The rise in colistin use renews concerns regarding the well-known potential for nephrotoxicity. Recent reports have described higher incidence of acute kidney injury (AKI) in patients treated with colistin, but most have small sample sizes, are not population-based, or study only one type of GNI $[5,6]$. AKI occurs in approximately $25-50 \%$ of patients treated with colistin [7-10], but has been reported as high as $90 \%$ in certain patients [11]. Those with comorbid conditions, including existing kidney disease, have increased risk of AKI while receiving colistin $[7,12]$. AKI post-colistin use often resolves [13], but increased risk of developing chronic kidney disease (CKD) after occurrence of AKI due to colistin use has been reported, with older age being a possible contributing factor [14].

The objectives of this study were to assess AKI and in-hospital mortality among patients hospitalized for severe GNIs who were receiving colistin or a new $\beta$-lactam/ $\beta$-lactamase inhibitor $(\beta L+\beta L I)$, stratified by the presence of baseline renal disease. The population-attributable risk of AKI due to colistin was used to estimate the potential amount of AKI that could be prevented by using a newer antibiotic in place of colistin.

\section{METHODS}

\section{Data Source}

The Premier Healthcare Database (PHD) was used for this analysis, with data from January 
2016 through December 2018. The PHD contains data from standard hospital discharge files, including patient demographics and disease states, insurance type, admission and discharge diagnoses, admission source and type, and discharge status. This database represents approximately $25 \%$ of US discharges from geographically diverse hospitals.

This study was performed in accordance with the Helsinki Declaration of 1964 and its later amendments. Institutional Review Board approval was not required based on the decision tool from the US National Institution of Health assessing a human subject study (https://grants. nih.gov/policy/humansubjects/hs-decision. htm).

\section{Study Design and Population}

This is a retrospective cohort study which evaluated the incidence of AKI and in-hospital mortality among patients with GNIs treated with colistin compared to propensity scorematched patients treated with $\beta \mathrm{L}+\beta \mathrm{LI}$ antibiotic agents.

Patients were included if they met all of the following criteria: (1) aged 18 + years with an inpatient stay between January 2016 and December 2018 (the 'index' period); (2) positive for a GNI; and (3) received at least 3 days of treatment with colistin (intravenous) or a $\beta \mathrm{L}+\beta \mathrm{LI}$ antibiotic agent. The $\beta \mathrm{L}+\beta \mathrm{LI}$ antibiotic agents included ceftazidime/avibactam, ceftolozane/tazobactam, or meropenem/vaborbactam. Imipenem/relabactam was not yet commercially available.

Patients were excluded for any of the following reasons: (1) evidence of cystic fibrosis or acute renal failure (ARF) present on admission by ICD-9/10 codes; (2) evidence of a dialysis procedure, defined using billing codes prior to the first dose of colistin or $\beta \mathrm{L}+\beta \mathrm{LI}$; and (3) received both colistin and a $\beta \mathrm{L}+\beta \mathrm{LI}$ antibiotic during the inpatient stay for $>72 \mathrm{~h}$ (Table S1).

\section{Patient and Hospital Characteristics}

Patient demographics included age, sex, race, and payer type. The Charlson-Deyo
Comorbidity Index (CCI) $[15,16]$ was calculated using ICD-9/10 codes. Prior exposure to other treatments that predispose to renal injury before the first dose of colistin or $\beta \mathrm{L}+\beta \mathrm{LI}$ agents was also examined (Table S2). An intensive care unit (ICU) stay or ventilation during hospitalization prior to the first dose of colistin or $\beta L+\beta L I$ agent, as well as sepsis present on admission or any inpatient hospitalization in the prior 30 days, was assessed. Hospital characteristics included geographic region, bed size, teaching status (yes/no), and location (urban/ rural).

\section{Identification of Gram-Negative Infections, Pathogens and Treatments}

All GNIs were confirmed by a positive culture from laboratory pathogen data or identification through pathogen susceptibility data. Information regarding infection site (grouped as respiratory, urinary, or bloodstream sources), susceptibility to carbapenems, and the type of pathogen [Enterobacterales, non-glucose fermenter, or other Gram-negative (GN) bacteria] were noted. The index date was the date of the first culture sample with a GN, CR pathogen. If a patient did not have any CR cultures identified, then the first carbapenem-sensitive GN culture became the reference culture and its associated index date.

Patients were classified as receiving colistin or one of the new $\beta L+\beta L I$ antibiotic agents active against GNIs, including ceftazidime-avibactam, ceftolozane-tazobactam, or meropenem-vaborbactam.

\section{Acute Kidney Injury, Acute Renal Failure, and In-Hospital Mortality Outcomes}

The incidence of ARF during treatment with colistin or $\beta L+\beta L I$ was defined using ICD-10 codes (Table S1). Incident AKI was defined as a dialysis procedure or ARF with or without a dialysis procedure (Table 1). In-hospital mortality was assessed during the index hospitalization. 
Table 1 Cohort attrition stratified by treatment regimen

\begin{tabular}{llc}
\hline & $\begin{array}{l}\text { Colistin }^{\mathbf{a}} \\
\boldsymbol{n}=\mathbf{6 8 1 1}\end{array}$ & $\begin{array}{l}\boldsymbol{\beta} \mathbf{+}+\boldsymbol{\beta L I} \text { agent } \\
\boldsymbol{n}=\mathbf{7 9 5 2}\end{array}$ \\
\hline Patients $\geq 18$ years of age & $6505(95.5 \%)$ & $7891(99.2 \%)$ \\
Inpatient hospitalization & $6434(94.5 \%)$ & $7654(96.3 \%)$ \\
Non-cystic fibrosis patients & $4499(66.1 \%)$ & $7235(91.0 \%)$ \\
Patients receiving $\geq 3$ days of treatment & $3320(48.7 \%)$ & $5713(71.8 \%)$ \\
Patients w/any microbiological results & $734(10.8 \%)$ & $1520(19.1 \%)$ \\
Patients w/confirmed Gram negative pathogen & $649(9.5 \%)$ & $1267(15.9 \%)$ \\
Patients w/no evidence of ARF POA or dialysis prior to 1st dose & $319(4.7 \%)$ & $657(8.3 \%)$ \\
Propensity score matched 1:1 & $256(3.8 \%)$ & $256(3.2 \%)$ \\
\hline
\end{tabular}

$A R F$ acute renal failure, $P O A$ present on admission

${ }^{\text {a }}$ Starting with patients receiving $\geq 1$ day of treatment

\section{Statistical Analysis}

Risk factors were identified a priori to assess differences in demographic and clinical characteristics between the colistin and $\beta \mathrm{L}+\beta \mathrm{LI}$ treatment groups, and to identify potential confounders and matching variables. A propensity score-matching approach was used to account for variables which may confound the relationship between treatments (colistin and $\beta \mathrm{L}+\beta \mathrm{LI}$ agents) and AKI.

Propensity scores were generated by logistic regression with treatment (colistin vs. any of the $\beta L+\beta L I$ agents) as the dependent variable. The independent variables in the model are shown in Table 2 . The matching algorithm used a caliper of 0.2 times the standard deviation of the logit of the propensity score to produce a 1:1 match, and the absolute standardized difference was used to compare characteristics between the colistin and $\beta \mathrm{L}+\beta \mathrm{LI}$ treatment groups post-match. In-hospital mortality was assessed during the index hospitalization and defined as death occurring in the hospital any time after initiating antibiotic therapy of interest. SAS v.9.3 (Cary, NC, USA) was used for all statistical analyses.

The rates of AKI and in-hospital mortality post-initiation of treatment were compared between patients receiving colistin or $\beta \mathrm{L}+\beta \mathrm{LI}$ after matching. P-values were calculated for differences in the rates of AKI overall and by baseline renal disease status using the Chisquare test. The rates of AKI and in-hospital mortality post-treatment initiation were compared between propensity score-matched treatment groups using logistic regression models to calculate adjusted odds ratios (ORs) while controlling for covariates. These analyses were additionally stratified by baseline renal disease, defined by the individual component of the CCI $[15,16]$. Associations of AKI with in-hospital mortality were also examined after stratifying by treatment (colistin vs. $\beta \mathrm{L}+\beta \mathrm{LI}$ agent). Since interaction was observed between treatment group and AKI, logistic regression models included joint effects. The population attributable risk percentage (PAR\%) of ARF due to colistin use was estimated using national projected utilization data from the Premier database and the risk difference of AKI incidence. We used Levin's formula to calculate the PAR\%.

\section{RESULTS}

\section{Attrition and Pathogen Characteristics}

After exclusion criteria were applied, 319 (4.7\%) patients were eligible for propensity score matching in the colistin group, and a total of 
Table 2 Patient, infection, and hospital characteristics pre- and post-propensity score matches

\begin{tabular}{|c|c|c|c|c|c|}
\hline & \multicolumn{2}{|c|}{ Unmatched sample } & \multicolumn{2}{|c|}{ 1:1 PSM sample } & \multirow[t]{2}{*}{$S^{a}$} \\
\hline & $\begin{array}{l}\text { Colistin } \\
n=319\end{array}$ & $\begin{array}{l}\beta \mathrm{LL}+\beta \mathrm{LI} \text { agent } \\
n=657\end{array}$ & $\begin{array}{l}\text { Colistin } \\
n=256\end{array}$ & $\begin{array}{l}\beta \mathrm{L}+\beta \mathrm{LI} \text { agent } \\
n=256\end{array}$ & \\
\hline \multicolumn{6}{|l|}{ Patient } \\
\hline Age, mean $\pm S D$ & $58.7 \pm 17.4$ & $61.2 \pm 15.6$ & $59.7 \pm 16.8$ & $59.2 \pm 15.2$ & $3 \%$ \\
\hline \multicolumn{6}{|l|}{ Sex } \\
\hline Female & $136(43 \%)$ & $283(43 \%)$ & $102(40 \%)$ & $106(41 \%)$ & $3 \%$ \\
\hline Male & $183(57 \%)$ & $374(57 \%)$ & $154(60 \%)$ & $150(59 \%)$ & $3 \%$ \\
\hline \multicolumn{6}{|l|}{ Race } \\
\hline White & $254(80 \%)$ & $462(70 \%)$ & $198(77 \%)$ & $194(76 \%)$ & $4 \%$ \\
\hline Non-White & $65(20 \%)$ & $195(30 \%)$ & $58(23 \%)$ & $62(24 \%)$ & $4 \%$ \\
\hline \multicolumn{6}{|l|}{ Payer type } \\
\hline Commercial & $32(10 \%)$ & $92(14 \%)$ & $28(11 \%)$ & $20(8 \%)$ & $11 \%$ \\
\hline Medicaid & $55(17 \%)$ & $112(17 \%)$ & $45(18 \%)$ & $49(19 \%)$ & $4 \%$ \\
\hline Medicare & $222(70 \%)$ & $434(66 \%)$ & $174(68 \%)$ & $178(70 \%)$ & $3 \%$ \\
\hline Other & $10(3 \%)$ & $19(3 \%)$ & $9(4 \%)$ & $9(4 \%)$ & $0 \%$ \\
\hline $\mathrm{CCI}$, mean $\pm \mathrm{SD}$ & $2.68 \pm 2.22$ & $2.75 \pm 2.29$ & $2.63 \pm 2.14$ & $2.76 \pm 2.08$ & $6 \%$ \\
\hline \multicolumn{6}{|l|}{ CCI categories } \\
\hline Renal disease (CCI) & $56(18 \%)$ & $140(21 \%)$ & $45(18 \%)$ & $49(19 \%)$ & $4 \%$ \\
\hline Diabetes w/complications (CCI) & $86(27 \%)$ & $182(28 \%)$ & $68(27 \%)$ & $70(27 \%)$ & $2 \%$ \\
\hline Diabetes w/o complications (CCI) & $34(11 \%)$ & $106(16 \%)$ & $30(12 \%)$ & $32(13 \%)$ & $2 \%$ \\
\hline Chronic pulmonary disease (CCI) & $153(48 \%)$ & $215(33 \%)$ & $115(45 \%)$ & $118(46 \%)$ & $2 \%$ \\
\hline Hospitalization in prior 30 days & $97(30 \%)$ & $206(31 \%)$ & $75(29 \%)$ & $85(33 \%)$ & $8 \%$ \\
\hline ICU prior to 1 st dose & $164(51 \%)$ & $283(43 \%)$ & $133(52 \%)$ & $139(54 \%)$ & $5 \%$ \\
\hline Exposure ARF drugs prior to 1 st dose & $271(85 \%)$ & $564(86 \%)$ & $218(85 \%)$ & $224(88 \%)$ & $7 \%$ \\
\hline ARF in Prior 3 months & $42(13 \%)$ & $116(18 \%)$ & $36(14 \%)$ & $35(14 \%)$ & $1 \%$ \\
\hline Ventilator prior to 1 st dose & $169(53 \%)$ & $222(34 \%)$ & $129(50 \%)$ & $132(52 \%)$ & $2 \%$ \\
\hline Sepsis present on admission & $172(54 \%)$ & $333(51 \%)$ & $140(55 \%)$ & $140(55 \%)$ & $0 \%$ \\
\hline \multicolumn{6}{|l|}{ Index infection } \\
\hline \multicolumn{6}{|l|}{ Infection site } \\
\hline Blood & $20(6 \%)$ & $59(9 \%)$ & $16(6 \%)$ & $15(6 \%)$ & $2 \%$ \\
\hline IAI & $1(0.3 \%)$ & $9(1 \%)$ & $0(0 \%)$ & $0(0 \%)$ & - \\
\hline Other & $18(6 \%)$ & $64(10 \%)$ & $17(7 \%)$ & $16(6 \%)$ & $2 \%$ \\
\hline Respiratory & $207(65 \%)$ & $195(30 \%)$ & $152(59 \%)$ & $152(59 \%)$ & $0 \%$ \\
\hline
\end{tabular}


Table 2 continued

\begin{tabular}{|c|c|c|c|c|c|}
\hline & Unmatched & Imple & 1:1 PSM $s$ & & $\overline{S D^{a}}$ \\
\hline & $\begin{array}{l}\text { Colistin } \\
n=319\end{array}$ & $\begin{array}{l}\beta \mathrm{L}+\beta \mathrm{LI} \text { agent } \\
n=657\end{array}$ & $\begin{array}{l}\text { Colistin } \\
n=256\end{array}$ & $\begin{array}{l}\beta \mathrm{L}+\beta \mathrm{LI} \text { agent } \\
n=256\end{array}$ & \\
\hline Skin & $21(7 \%)$ & $120(18 \%)$ & $20(8 \%)$ & $17(7 \%)$ & $5 \%$ \\
\hline Urine & $52(16 \%)$ & $210(32 \%)$ & $51(20 \%)$ & $56(22 \%)$ & $5 \%$ \\
\hline Pathogen & & & & & \\
\hline Enterbacterales & $82(26 \%)$ & $257(39 \%)$ & $72(28 \%)$ & $80(31 \%)$ & $7 \%$ \\
\hline Non-fermenter & $236(74 \%)$ & $389(59 \%)$ & $183(71 \%)$ & $175(68 \%)$ & $7 \%$ \\
\hline Other G-bacteria & $1(0.3 \%)$ & $11(2 \%)$ & $1(0.4 \%)$ & $1(0.4 \%)$ & $0 \%$ \\
\hline Carbapenem suscep & & & & & \\
\hline Not susceptible & $225(71 \%)$ & $489(74 \%)$ & $184(72 \%)$ & $179(70 \%)$ & $4 \%$ \\
\hline Not tested & $18(6 \%)$ & $44(7 \%)$ & $14(5 \%)$ & $20(8 \%)$ & $9 \%$ \\
\hline Susceptible & $76(24 \%)$ & $124(19 \%)$ & $58(23 \%)$ & $57(22 \%)$ & $1 \%$ \\
\hline Hospital & & & & & \\
\hline Bed size & & & & & \\
\hline$<300$ & $107(34 \%)$ & $171(26 \%)$ & $86(34 \%)$ & $86(34 \%)$ & $0 \%$ \\
\hline $300-499$ & $97(30 \%)$ & $149(23 \%)$ & $73(29 \%)$ & $74(29 \%)$ & $1 \%$ \\
\hline $500+$ & $115(36 \%)$ & $337(51 \%)$ & $97(38 \%)$ & $96(38 \%)$ & $1 \%$ \\
\hline Teaching facility & $156(49 \%)$ & $406(62 \%)$ & $128(50 \%)$ & $130(51 \%)$ & $2 \%$ \\
\hline Region & & & & & \\
\hline Midwest & $99(31 \%)$ & $245(37 \%)$ & $86(34 \%)$ & $93(36 \%)$ & $6 \%$ \\
\hline Northeast & $29(9 \%)$ & $45(7 \%)$ & $21(8 \%)$ & $25(10 \%)$ & $5 \%$ \\
\hline South & $174(55 \%)$ & $325(49 \%)$ & $133(52 \%)$ & $126(49 \%)$ & $5 \%$ \\
\hline West & $17(5 \%)$ & $42(6 \%)$ & $16(6 \%)$ & $12(5 \%)$ & $7 \%$ \\
\hline Location & & & & & \\
\hline Rural & $65(20 \%)$ & $48(7 \%)$ & $40(16 \%)$ & $37(14 \%)$ & $3 \%$ \\
\hline Urban & $254(80 \%)$ & 609 (93\%) & $216(84 \%)$ & $219(86 \%)$ & $3 \%$ \\
\hline
\end{tabular}

$A R F$ acute renal failure, $C C I$ Charlson Comorbidity Index, $I A I$ intra-abdominal infection, $I C U$ intensive care unit, $S D$ standard deviation

${ }^{a}$ Absolute standardized difference for 1:1 propensity score-matched sample

$657(8.3 \%)$ for $\beta L+\beta L I$ agents (Table 1). Patient counts were consistently higher for the $\beta \mathrm{L}+\beta \mathrm{LI}$ group following application of each restriction, particularly after CF patients were excluded $(66.1 \%$ vs. $91.0 \%)$. No patients receiving meropenem-vaborbactam were part of the matched sample (Table 1).

In the propensity matched sample $(n=256$ in each treatment group), for both the colistinand $\beta \mathrm{L}+\beta \mathrm{LI}$ treated groups, the distribution 


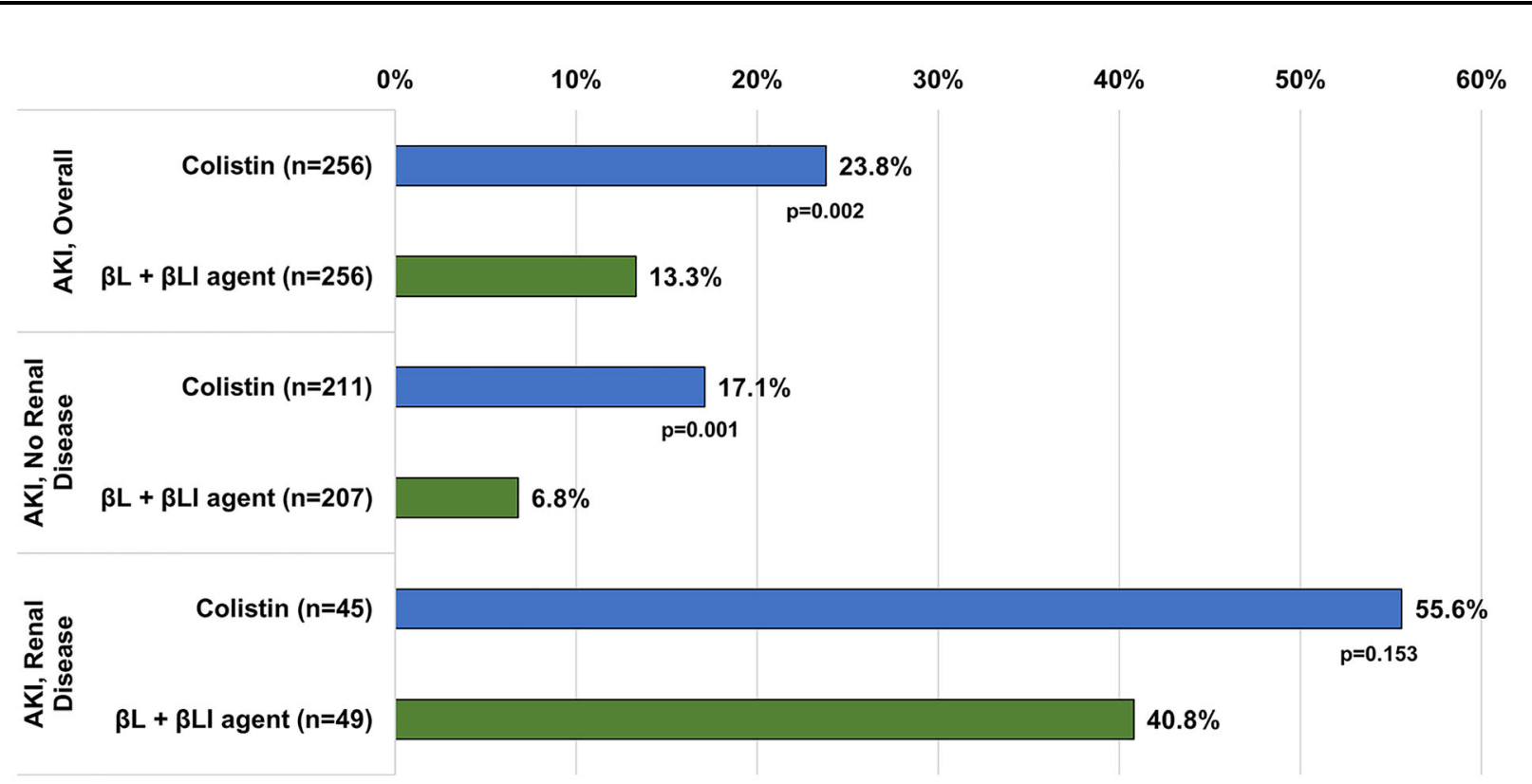

Fig. 1 Rates of AKI by treatment group and baseline renal disease status among propensity score-matched sample

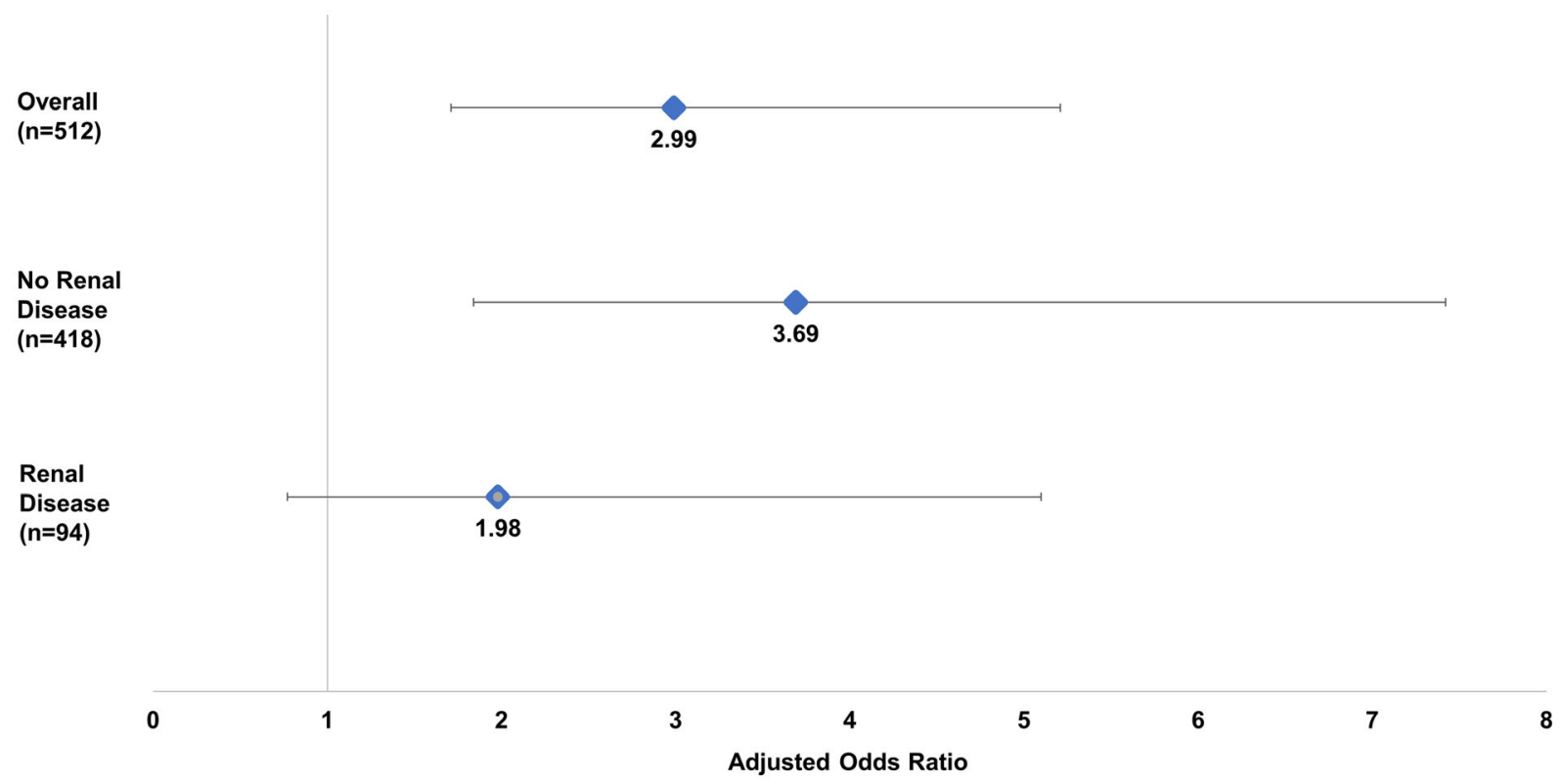

Fig. 2 Adjusted odds of AKI for colistin versus $\beta L+\beta L I$ agents, stratified by baseline renal disease, among propensity score-matched sample

between CRE and non-fermenter pathogens was similar, $28 \%$ vs. $31 \%$ and $71 \%$ vs. $68 \%$ respectively (Table 2). The most frequently treated pathogen was Pseudomonas aeruginosa $(n=96$, $37.5 \%$ vs. $n=157,61.3 \%)$. The actual distribution of specific pathogens is shown in Table S3.

\section{Patient and Hospital Characteristics}

Table 2 displays baseline characteristics for the colistin and $\beta \mathrm{L}+\beta \mathrm{LI}$ treatment groups, and preand post-propensity score matching. Overall, 
Table 3 Adjusted mortality risk for colistin versus new agents among propensity score-matched sample, without accounting for joint effects of treatment and AKI

\begin{tabular}{lcccc}
\hline $\begin{array}{l}\text { Final stepwise model: treatment and AKI forced into } \\
\text { model }^{\mathbf{a}}\end{array}$ & $\begin{array}{l}\text { OR } \\
\text { estimate }\end{array}$ & $\begin{array}{l}\text { Lower 95\% } \\
\text { CI }\end{array}$ & $\begin{array}{l}\text { Upper 95\% } \\
\text { CI }\end{array}$ & $\boldsymbol{p}$ value \\
\hline Ventilated at ANY point & 13.80 & 5.02 & 37.88 & $<0.0001$ \\
Age & 1.03 & 1.01 & 1.06 & 0.006 \\
Mild liver disease & 2.65 & 0.96 & 7.33 & 0.060 \\
Malignancy & 12.53 & 5.04 & 31.15 & $<0.0001$ \\
Treatment colistin vs. new agents & 1.36 & 0.74 & 2.51 & 0.318 \\
AKI yes vs. no & 3.81 & 2.00 & 7.27 & $<0.0001$ \\
\hline
\end{tabular}

$A K I$ acute kidney injury

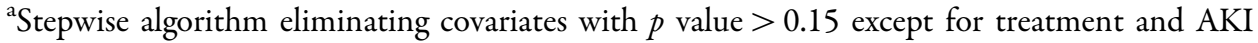

the matched cohorts were similar for patient, infection site and hospital characteristics.

The most common comorbid conditions were chronic pulmonary disease ( $45 \%$ vs. $46 \%)$ and diabetes with and without complications (39\% vs. $40 \%$ ). Baseline renal disease was present in $18 \%$ of the colistin and $19 \%$ of the $\beta \mathrm{L}+\beta \mathrm{LI}$ group.

\section{Associations of Treatments with AKI}

Overall, AKI had developed in $23.8 \%$ of the colistin and $13.3 \%$ of the $\beta L+\beta L I$ group (Fig. 1). Following post-propensity score matching, patients without baseline renal disease $(n=418)$ experienced AKI during hospitalization to a higher degree in the colistin group compared to the $\beta \mathrm{L}+\beta \mathrm{LI}$ group $(17.1 \%$ vs. $6.8 \%)$. Among patients with baseline renal disease, there was no statistically significant difference between the groups.

After additional adjustment with logistic regression, among the propensity score-matched sample, patients receiving colistin had approximately threefold higher odds $(\mathrm{OR}=3.0$; $95 \% \mathrm{CI} 1.71,5.21)$ of $\mathrm{AKI}$ versus $\beta \mathrm{L}+\beta \mathrm{LI}$ agents (Fig. 2). Baseline renal disease $(\mathrm{OR}=5.1 ; 95 \% \mathrm{CI}$ $2.70,9.62)$ was also a significant independent risk factor for AKI (data not shown). Stratified analysis revealed that, among patients without existing renal disease $(n=418)$, colistin use was associated with 3.7 higher odds of AKI versus $\beta \mathrm{L}+\beta \mathrm{LI}$ agents (95\% CI 1.84, 7.42) (Fig. 2). In contrast, among those with existing renal disease $(n=94)$, odds of AKI were not statistically different between the colistin and $\beta \mathrm{L}+\beta \mathrm{LI}$ agent treatment groups (OR $=2.0 ; 95 \%$ CI 0.77 . 5.10).

\section{Associations of Treatments with In- hospital Mortality}

In-hospital mortality was higher in the colistin group compared to the $\beta \mathrm{L}+\beta \mathrm{LI}$ group $(15.2 \%$ vs. $10.5 \%$ ). While adjusted odds of inpatient mortality for colistin versus $\beta \mathrm{L}+\beta \mathrm{LI}$ treatment was not statistically significant, occurrence of AKI during the inpatient visit was associated with almost four-fold higher odds of mortality compared to those without AKI (OR $=3.8 ; 95 \%$ CI 2.00, 7.27) (Table 3). The joint risk of mortality among patients receiving colistin and having AKI was more than 3 times that of patients receiving new agents with AKI $(\mathrm{OR}=3.23$, 95\% CI 1.09, 9.52) (Table 4). Similarly, adjusted odds of mortality for patients with AKI treated with colistin was six times that of colistin patients without AKI $(\mathrm{OR}=6.13$; $95 \%$ CI 2.53, 14.71). Conversely, among patients on new agents, no association was observed between AKI and mortality. 
Table 4 Risk of mortality among propensity score-matched sample, colistin vs. new agents by AKI status

\begin{tabular}{|c|c|c|c|c|}
\hline $\begin{array}{l}\text { Final stepwise model-joint effect of AKI and treatment } \\
\text { type } \mathrm{e}^{\mathrm{a}}\end{array}$ & $\begin{array}{l}\text { OR } \\
\text { estimate }\end{array}$ & $\begin{array}{l}\text { Lower } 95 \% \\
\text { CI }\end{array}$ & $\begin{array}{l}\text { Upper } 95 \% \\
\text { CI }\end{array}$ & $p$ value \\
\hline Ventilated at ANY point & 15.87 & 5.69 & 44.23 & $<0.0001$ \\
\hline Age & 1.03 & 1.01 & 1.06 & 0.0056 \\
\hline Rheumatologic disease & 4.81 & 0.67 & 34.78 & 0.1196 \\
\hline Mild liver disease & 3.01 & 1.08 & 8.44 & 0.0358 \\
\hline Malignancy & 12.95 & 5.16 & 32.49 & $<0.0001$ \\
\hline New agent AKI vs. new agent no AKI & 1.67 & 0.60 & 4.67 & 0.3312 \\
\hline Colistin no AKI vs. new agent no AKI & 0.88 & 0.41 & 1.90 & 0.7415 \\
\hline Colistin AKI vs. new agent no AKI & 5.37 & 2.31 & 12.49 & $<0.0001$ \\
\hline Colistin AKI vs. new agent $\mathrm{AKI}^{\mathrm{b}}$ & 3.23 & 1.09 & 9.52 & 0.0347 \\
\hline Colistin AKI vs. colistin no $\mathrm{AKI}^{\mathrm{b}}$ & 6.13 & 2.53 & 14.71 & $<0.0001$ \\
\hline
\end{tabular}

$A K I$ acute kidney injury

${ }^{a}$ Stepwise algorithm eliminating covariates with $p$ value $>0.15$ except for treatment and AKI

${ }^{\mathrm{b}}$ Not a model coefficient

\section{Population Attributable Risk of Colistin in AKI}

Based on the calculated PAR\%, we estimated that, at the end of $2018,28.6 \%$ of AKI in the population treated with either colistin or a $\beta \mathrm{L}+\beta \mathrm{LI}$ agent for a GNI could have been avoided by not using colistin. We estimated the proportion of use in 2018 of colistin to be $23 \%$ of the total of both colistin and $\beta \mathrm{L}+\beta \mathrm{LI}$ agents used for GNIs and used the OR of 3.0 of the risk of AKI for colistin versus $\beta \mathrm{L}+\beta \mathrm{LI}$ agents as an approximation of the relative risk. This assumes that colistin and $\beta \mathrm{L}+\beta \mathrm{LI}$ agents were used only to treat CR GNIs. If the $\beta \mathrm{L}+\beta \mathrm{LI}$ group was used more empirically with some infections being found to be Gram-positives or pathogens less likely to be treated with colistin, then the true proportion of colistin use would be greater. This would make our estimate a lower bound estimate.

\section{DISCUSSION}

The use of colistin has increased in recent years along with the incidence of CR GNIs [2], renewing concerns over colistin-associated nephrotoxicity. In this retrospective cohort study, 512 patients with GNIs were matched on baseline characteristics. There was an increased risk of AKI in patients treated with colistin $(23.8 \%)$ versus newer $\beta \mathrm{L}+\beta \mathrm{LI}$ antibiotics $(13.3 \%)$ that may have equal or better coverage for CR GNIs. These findings corroborate prior studies supporting a link between colistin use and incident AKI $[9,13]$. A recent meta-analysis identified 5 randomized controlled trials comparing nephrotoxicity of colistin to minimally nephrotoxic antibiotics. The authors estimated a colistin-associated nephrotoxicity rate of $36 \%$, and $140 \%$ increased risk, compared to $\beta L$-based regimens [17]. We estimated that the percentage of AKI attributable to colistin use in 2018 was $25-29 \%$, suggesting that roughly 1 in 4 incident cases of AKI may be prevented by utilizing newer, less toxic antibiotics. To our knowledge, no previous studies have reported the PAR\% for AKI due to colistin. This finding 
may have significant implications for healthcare costs, and morbidity and mortality associated with colistin use.

Elevated risk of AKI was observed among patients without pre-existing renal disease who were treated with colistin compared to those treated with $\beta L+\beta L I$. Among patients with renal disease at baseline, $56 \%$ of colistin- and $41 \%$ of $\beta \mathrm{L}+\beta \mathrm{LI}$-treated patients had subsequent AKI, although this difference was not significant, possibly due to the relatively small sample size of patients with renal disease. Several studies have documented significantly higher risk of AKI among patients with existing renal disease or chronic kidney disease [7, 18]. To our knowledge, colistin-associated AKI has not been studied specifically among those without existing renal disease. It is possible that clinicians who prescribe colistin to patients who have pre-existing renal disease may take additional precautions to prevent incident AKI.

The current study demonstrates that, among those who develop AKI, patients treated with colistin have threefold greater adjusted odds of mortality compared to $\beta \mathrm{L}+\beta \mathrm{LI}$. This aligns with previous studies that reported colistin use to treat GNIs was associated with an increased risk of mortality. A meta-analysis estimated the odds of 30-day mortality at 1.5 times higher for patients receiving colistin versus $\beta \mathrm{L}+\beta \mathrm{LI}$ antibiotics [13], a result not inconsistent with the point estimate of 1.36 in this study, without taking into consideration the joint effect of AKI and treatment. Moreover, a prospective observational study of 99 patients receiving colistin and 38 patients receiving ceftazidime-avibactam found that patients receiving colistin had a significantly higher rate of 30-day in-hospital mortality (32\% vs. $9 \%)$, although this was limited to treatment of CRE [19].

Timing of AKI in colistin-treated patients may play a role in mortality risk, with earlystage AKI (within 7 days of first colistin dose) conferring more than fourfold higher odds of mortality compared to later-stage AKI [20]. In our study, patients receiving colistin who developed AKI had approximately 6 times the odds of mortality compared to those without AKI, while odds of mortality among patients treated with $\beta \mathrm{L}+\beta \mathrm{LI}$ were not significantly higher for those with AKI versus no AKI. Prior research has demonstrated that, among patients on colistin with AKI, those whose AKI resolved had better survival compared to those whose AKI did not ( $88 \%$ vs. $53 \%, p=0.001)$ [21]. The current study is consistent with previous findings, with higher mortality in the colistin treatment group compared to the $\beta \mathrm{L}+\beta \mathrm{LI}$ treatment group, and, among patients on colistin, greater odds of mortality associated with incident AKI. Patients prescribed colistin in the context of AKI may have more unidentified adverse characteristics, leading their physicians to use colistin despite their AKI. Additional research is indicated to address this question.

Our study's findings should be interpreted in the context of its strengths and limitations. Strengths include that these data are real-world from a large US hospital discharge database. Some prior studies were not population-based, only included one or two GN pathogens, or did not adjust for confounders. We controlled for selection bias using propensity-score techniques to match treatment groups on measured variables, as well as multiple logistic regression. The current study was agnostic in terms of pathogen, although propensity score matching controlled for Enterobacterales versus nonfermenters. More patients with Acinetobacter infection were treated with colistin than new agents. As Acinetobacter spp. are known to be associated with higher mortality than other GN pathogens [22], this was included as an indicator variable in our mortality regression model and did not alter the results.

Limitations include that this analysis utilizes an administrative dataset with an observational retrospective approach with the possibility of selection bias and misclassification. If a patient was admitted with high risk for AKI not noted in the index hospitalization, there may have been a higher probability of being treated with a $\beta L+\beta L I$ antibiotic. Similarly, not every nephrotoxic agent used prior to treatment would necessarily be identified, but any bias would be in the direction of the $\beta L+\beta L I$ antibiotics; clinicians normally refrain from using additional nephrotoxic agents such as colistin. This would result in a conservative bias away from an association of colistin with AKI or 
mortality. We also could not time the diagnosis of AKI during the hospitalization. Some incident AKI may have occurred prior to treatment but after admission, resulting in misclassification. This would also result in a bias away from a colistin association with AKI. Severity could be a potential confounder. We do not have an index of severity in Premier data; rather, we used a surrogate measure, the presence of mechanical ventilation, that may not be as granular as a severity score. Laboratory results, if available, would have improved the classification of a new AKI diagnosis. However, others have shown the validity of using administrative codes for classifying incident AKI in an observational setting $[23,24]$. Higher doses of colistin have been associated with increased risk of AKI [25], but we were unable to evaluate dosing differences.

\section{CONCLUSIONS}

Patients receiving colistin for GNIs had significantly higher odds of developing AKI compared to those on $\beta \mathrm{L}+\beta \mathrm{LI}$ antibiotics, including patients without baseline renal disease. Among patients on colistin, there were significantly higher odds of mortality for those who developed AKI versus those who did not, which was not observed among $\beta \mathrm{L}+\beta \mathrm{LI}$-treated patients. Further research is warranted on the nephrotoxicity of colistin in real-world settings. Our findings suggest that almost 1 in 4 incident cases of AKI may be prevented by utilizing more recently developed, less toxic antibiotics. The implications for healthcare costs, morbidity, and mortality also merit future investigations.

\section{ACKNOWLEDGEMENTS}

Funding. This work and the Rapid Service Fee were supported by Shionogi, Inc, Florham Park, NJ, USA. Employees of the study sponsor were involved in the study design, as well as collection, analysis, interpretation of the data, and in critically revising the manuscript for important intellectual content. No funding to the authors was provided for the preparation of the manuscript.

Authorship. All named authors meet the International Committee of Medical Journal Editors (ICMJE) criteria for authorship for this article, take responsibility for the integrity of the work as a whole, and have given their approval to publish this version. All authors read and approved the final manuscript.

Author Contributions. Casey Doremus and Stephen W Marcella conceived and designed the study, performed the analysis, contributed to data interpretation, and critically revised the manuscript for intellectual content. Roger M Echols and Bin Cai critically revised the manuscript.

Medical Writing/Editorial Assistance. Medical writing support was provided by Eve Hunter-Featherstone and Jenifer Wogen at Genesis Research. Funding for this service was provided by Shionogi Inc.

Prior Presentation. A subset of the findings in this study were presented as a poster at the virtual European Congress of Clinical Microbiology and Infectious Diseases (ECCMID) in 2020 .

Disclosures. Casey Doremus, Stephen W Marcella, and Bin Cai are employees of Shionogi, Inc., Florham Park, NJ USA, and Roger M Echols is a consultant for Shionogi, Inc.

Compliance with Ethics Guidelines. This study was conducted using an anonymous, publicly available secondary dataset that meets the US HIPAA requirement. Ethics approval and consent to participate are not applicable for the current study per US 45 Code of Federal Regulations Part 46, human participant protection regulations, and Protections of Human Subjects.

This study was performed in accordance with the Helsinki Declaration of 1964, and its later amendments. Analysis of commercially available de-identified secondary data sources is considered exempt from the requirements for "human subjects research" in the US. Since this 
study utilized only de-identified data and did not involve collection, use or transmittal of individually identifiable data, Institutional Review Board (IRB) approval was not required.

Data Availability. The data that support the findings of this study are available from Premier Inc., but restrictions apply to the availability of these data, which were used under license for the current study, and so are not publicly available. Data are, however, available from the authors upon reasonable request and with permission of Premier Inc.

Open Access. This article is licensed under a Creative Commons Attribution-NonCommercial 4.0 International License, which permits any non-commercial use, sharing, adaptation, distribution and reproduction in any medium or format, as long as you give appropriate credit to the original author(s) and the source, provide a link to the Creative Commons licence, and indicate if changes were made. The images or other third party material in this article are included in the article's Creative Commons licence, unless indicated otherwise in a credit line to the material. If material is not included in the article's Creative Commons licence and your intended use is not permitted by statutory regulation or exceeds the permitted use, you will need to obtain permission directly from the copyright holder. To view a copy of this licence, visit http://creativecommons.org/licenses/by$\mathrm{nc} / 4.0 /$.

\section{REFERENCES}

1. Poirel L, Jayol A, Nordmann P. Polymyxins: antibacterial activity, susceptibility testing, and resistance mechanisms encoded by plasmids or chromosomes. Clin Microbiol Rev. 2017;30(2): 557-96.

2. Lim LM, Ly N, Anderson D, et al. Resurgence of colistin: a review of resistance, toxicity, pharmacodynamics, and dosing. Pharmacotherapy. 2010;30(12):1279-91.

3. World Health Organization. Global priority list of antibiotic resistant bacteria to guide research, discovery and development of new antibiotics. 2017.
4. US Department of Health and Human Services Center for Disease Control and Prevention. Antibiotic resistance threats in the United States. 2019.

5. Wunderink RG, Giamarellos-Bourboulis EJ, Rahav $G$, et al. Effect and safety of meropenem-vaborbactam versus best-available therapy in patients with carbapenem-resistant enterobacteriaceae infections: the TANGO II randomized clinical trial. Infect Dis Ther. 2018;7(4):439-55.

6. Motsch J, Murta-de-Oliveira C, Stus V, et al. RESTORE-IMI 1: a multicenter, randomized, double-blind trial comparing efficacy and safety of imipenem/relebactam vs. colistin plus imipenem in patients with imipenem-nonsusceptible bacterial infections. Clin Infect Dis. 2020;70(9):1799-808.

7. Durante-Mangoni E, Andini R, Signoriello S, et al. Acute kidney injury during colistin therapy: a prospective study in patients with extensively-drug resistant Acinetobacter baumannii infections. Clin Microbiol Infect. 2016;22(12):984-9.

8. Hassan MM, Gaifer Z, Al-Zakwani IS. Incidence and risk factors of nephrotoxicity in patients on colistimethate sodium. Int J Clin Pharm. 2018;40(2): 444-9.

9. Oliota AF, Penteado ST, Tonin FS, Fernandez-Llimos F, Sanches AC. Nephrotoxicity prevalence in patients treated with polymyxins: a systematic review with meta-analysis of observational studies. Diagn Microbiol Infect Dis. 2019;94(1):41-9.

10. Patek TM, Teng C, Kennedy KE, Alvarez CA, Frei CR. Comparing acute kidney injury reports among antibiotics: a pharmacovigilance study of the FDA adverse event reporting system (FAERS). Drug Saf. 2020;43(1):17-22.

11. Thammathiwat $\mathrm{T}$, Tiranathanagul $\mathrm{K}$, Srisawat $\mathrm{N}$, Susantitaphong P, Praditpornsilpa K, Eiam-Ong S. Clinical and subclinical acute kidney injury in multidrug-resistant septic patients treated with colistimethate sodium: Incidence and clinical outcomes. Nephrology (Carlton). 2020;25(1):32-9.

12. Gunay E, Kaya S, Baysal B, Yuksel E, Arac E. Evaluation of prognosis and nephrotoxicity in patients treated with colistin in intensive care unit. Ren Fail. 2020;42(1):704-9.

13. Chien HT, Lin YC, Sheu CC, Hsieh KP, Chang JS. Is colistin-associated acute kidney injury clinically important in adults? A systematic review and metaanalysis. Int J Antimicrob Agents. 2020;55(3): 105889.

14. Meraz-Munoz A, Gomez-Ruiz I, Correa-Rotter R, Ramirez-Sandoval JC. Chronic kidney disease after acute kidney injury associated with intravenous 
colistin use in survivors of severe infections: a comparative cohort study. J Crit Care. 2018;44: 244-8.

15. Charlson ME, Pompei P, Ales KL, MacKenzie CR. A new method of classifying prognostic comorbidity in longitudinal studies: development and validation. J Chronic Dis. 1987;40(5):373-83.

16. Deyo RA, Cherkin DC, Ciol MA. Adapting a clinical comorbidity index for use with ICD-9-CM administrative databases. J Clin Epidemiol. 1992;45(6): 613-9.

17. Eljaaly K, Bidell MR, Gandhi RG, et al. Colistin nephrotoxicity: meta-analysis of randomized controlled trials. Open Forum Infect Dis 2021;8(2).

18. Min KL, Son ES, Kim JS, Kim SH, Jung SM, Chang MJ. Risk factors of colistin safety according to administration routes: intravenous and aerosolized colistin. PLoS ONE. 2018;13(11):e0207588.

19. van Duin D, Lok JJ, Earley M, et al. Colistin versus ceftazidime-avibactam in the treatment of infections due to carbapenem-resistant enterobacteriaceae. Clin Infect Dis. 2018;66(2):163-71.

20. Ko H, Jeon M, Choo E, et al. Early acute kidney injury is a risk factor that predicts mortality in patients treated with colistin. Nephron Clin Pract. 2011;117(3):c284-8.
21. Miano TA, Lautenbach E, Wilson FP, Guo W, Borovskiy Y, Hennessy S. Attributable risk and time course of colistin-associated acute kidney injury. Clin J Am Soc Nephrol. 2018;13(4):542-50.

22. Leão ACQ, Menezes PR, Oliveira MS, Levin AS. Acinetobacter spp. are associated with a higher mortality in intensive care patients with bacteremia: a survival analysis. BMC Infect Dis $2016 ; 16(1)$

23. Patel UD, Hardy NC, Smith DH, et al. Validation of acute kidney injury cases in the mini-sentinel distributed database. Mini-Sentinel, 2013.

24. Waikar SS, Wald R, Chertow GM, et al. Validity of international classification of diseases, ninth revision, clinical modification codes for acute renal failure. J Am Soc Nephrol. 2006;17(6):1688-94.

25. Almutairy R, Aljrarri W, Noor A, et al. Impact of colistin dosing on the incidence of nephrotoxicity in a tertiary care hospital in Saudi Arabia. Antibiotics. 2020;9(8):485. 